\title{
Cloning and Expression Analysis of a Farnesyl Diphosphate Synthase (FPPS) Gene from Chamaemelum nobile
}

\author{
Xiao-Meng $\mathrm{LIU}^{1}$, Ting-Ting $\mathrm{TAO}^{1}$, Xiang-Xiang MENG ${ }^{1}$, \\ Wei-Wei ZHANG ${ }^{1}$, Jie CHANG ${ }^{2,3}$, Feng XU ${ }^{1 *}$
}

\author{
${ }^{1}$ Yangtze University, College of Horticulture and Gardening, Jingzhou, Hubei 434025, China; LiuXM925@163.com; \\ 18010872961@163.com;dreamer9212@163.com;wwzhangchn@163.com;xufeng198@126.com (*correspondingauthor) \\ ${ }^{2}$ Hubei Collaborative Innovation Center of Targeted Antitumor Drug, Jingmen, Hubei 448000, China; 18986662379@163.com \\ ${ }^{3}$ College of Chemical Engineering and Pharmacy, Jingchu University of Technology, Jingmen, Hubei 448000, China
}

\begin{abstract}
Farnesyl diphosphate synthase (FPPS), an isopentenyl transferase, catalyzes the condensation reaction of five carbon isopentenyl pyrophosphate (IPP) and dimethylallyl pyrophosphate (DMAPP) to form fifteen carbon farnesyl pyrophosphate (FPP), which is the key precursor for sesquiterpene biosynthesis. In this study, a FPPS gene (CnFPPS) was cloned from Chamaemelum nobile. The full-length cDNA of CnFPPS is 1239 bp and contains an open reading frame (ORF) of 1029 bp encoding 342 amino acids. The theoretical molecular weight and $\mathrm{pI}$ of the CnFPPS protein are $39.38 \mathrm{kDa}$ and 5.59, respectively. Multiple alignment analysis showed the protein sequence of CnFPPS had a high homology with FPPS proteins from other plants. The deduced amino acid of CnFPPS contained five conservative domains such as substrate binding pocket, substrate- $\mathrm{Mg}^{2+}$ binding site, catalytic site, aspartate-rich region 1 and 2, suggesting CnFPPS is one member of FPPS family in C. nobile. Phylogenetic analysis based on the amino acid sequences of FPPSs showed that CnFPPS was closely related to the FPPS of Matricaria chamomilla. The result of qRT-PCR revealed that CnFPPS gene was constitutively expressed in different tissues of C. nobile, with the highest expression in the root. These findings improve the understanding of the synthesis and regulation of the terpenoid compounds at the molecular level and lay a foundation for studying the regulatory functions of CnFPPS in terpenoid biosynthetic pathway in C. nobile.
\end{abstract}

Keywords: expression profile, farnesyl diphosphate synthase, Roman chamomile, sequence analysis

\section{Introduction}

Chamaemelum nobile, commonly referred to as "Roman chamomile", a perennial herb belonging to the Asteraceae family native to Western Europe (Xiao, 2003), is a widely used medicinal plant due to its antioxidant and antiinflammatory effects (Ma et al., 2007). C. nobile is utilized to treat indigestion, appetite loss, nausea, vomiting, and other symptoms because it alleviates spasm and elicits antiinflammatory and sedative effects (Srivastava et al., 2010). C. nobile is rich in complex essential oils, and more than 100 kinds of components of essential oils have been identified from this plant (Fauconnier et al., 2010). The active chemical constituents of the essential oils include terpenoids, flavonoids, esters, etc.
Among these chemical constituents, terpenoids are the main active components (Farhoudi, 2013). Therefore, we can significantly improve the quality and therapeutic value of $C$. nobile by increasing the content of terpenoids.

Terpenoids or isoprenoids, the most abundant secondary metabolite in terms of chemical structure, are a class of compounds composed of isoprene units (Yue et al., 2011). Terpenoids are divided into the following groups based on the number of isoprene units: monoterpene (C10), sesquiterpene (C15), diterpene (C20), and triterpene (C30). More than 25,000 types of terpenoids have been identified in plants (Chang et al., 2015). Many terpenoids are utilized for economic and chemical applications, including flavors, pigments, waxes, rubbers, and pharmaceuticals such as vitamins, taxol and artemisinin (Liu et al., 2006). Terpenoids can also play an important 
role in the essential biological processes of plants such as growth, development, reproduction, and adaptation to environmental challenges (Yu et al., 2009). In higher plants, terpenoids are synthesized by the mevalonate (MVA) pathway in the cytoplasm and the 2-C-methyl-D-erythritol 4-phosphate (MEP) pathway in plastids (Vranová et al., 2012). The synthetic precursor of all terpenoid substances is isopentenyl pyrophosphate (IPP) (Enfissi et al., 2005). As an important intermediate in the downstream of MVA pathway, farnesyl pyrophosphate (FPP) is generated by combining dimethylallyl pyrophosphate (DMAPP) with IPP and various types of terpenoids are consequently produced by the action of different terpene synthases and further by the modification such as glycosylation and methylation (He et al., 2011; Ma et al., 2015).

Farnesyl diphosphate synthase (FPPS), a key ratelimiting enzyme in the biosynthesis pathway of terpenoids, catalyzes 1, 4-head-to-tail condensation reaction of DMAPP and IPP into FPP (Lange et al., 2000; Cornish, 1993). Many important secondary metabolites, such as terpene alcohols, sterols, ubiquinones, and carotenoids, are synthesized from FPP. Under the action of synthetic enzymes, FPP also generates various sesquiterpene compounds, such as $(-)-\alpha$-bisabolone oxide $A$ and $\alpha$ bisabolol (Delourme et al., 1994; Chappell et al., 1995), which are essential for plants and humans. For example, phytoalexins help regulate plant growth, development, and immunity. Sesquiterpene compounds, such as $\alpha$-bisabolol, induce anti-inflammatory, antibacterial, antispasmodic, sedative, analgesic, anticorrosion, antioxidant, and anticancer effects, and these compounds are important additives in several products, such as cosmetics and medicine (Sharkey et al., 2013). FPPS is one of the most widely examined isopentenyl transferase (Ohto et al., 1999). With the significance of this enzyme in isoprenoid metabolism, genes encoding FPPSs have been isolated and extensively characterized from many plants, including Artemisia annua (Matsushita et al., 1996), peppermint (Lange et al., 2000), cotton (Liu et al., 1997), white lupine (Attucci et al., 1995), and Arabidopsis thaliana (Delourme et al., 1994).

Although $C$. nobile is an important medicinal plant, enzymes or genes involved in the biosynthetic pathway of its main medicinal constituents sesquiterpene compounds have been rarely identified. To investigate the biosynthesis pathway of terpenoids in C. nobile, we should identify and characterize each gene involved in this pathway.

In this study, we report the cloning and characterization of a full-length cDNA of FPPS (CnFPPS) from $C$. nobile. We also examined the expression pattern in different tissues in C. nobile.

\section{Materials and Methods}

\section{Plantmaterial}

C. nobile was grown at $25 / 18^{\circ} \mathrm{C}$ in a growth chamber $(16$ $\mathrm{h} / 8 \mathrm{~h}$ light/dark). The leaves, stems, roots, and flowers of $C$. nobile were collected from the Botanical Garden at Yangtze University and immediately placed in a $-80^{\circ} \mathrm{C}$ until use. Primer synthesis and DNA sequencing were performed by Shanghai Sangon Biotechnology Company, China.

\section{Cloning of CnFPPS}

Total RNA was isolated from frozen plant tissues of $C$. nobile using the TaKaRaMiniBEST Plant RNA Extraction kit. The first-strand cDNA was synthesized using PrimeScript ${ }^{\mathrm{TM}}$ First-Strand cDNA Synthesis Kit according to manufacturer's instructions. The specific primers, namely, G1 and G2, (Table 1) as given below were designed based on the FPPS unigene sequence of $C$. nobile transcriptome data (GenBank accession number SRR4021832). CnFPPS cDNA was amplified under the following conditions: $94^{\circ} \mathrm{C}$ for $4 \mathrm{~min}$; 30 cycles of amplification at $94^{\circ} \mathrm{C}$ for $30 \mathrm{~s}, 61^{\circ} \mathrm{C}$ for $30 \mathrm{~s}$, and $72^{\circ} \mathrm{C}$ for 90 $s$; and a final extension at $72{ }^{\circ} \mathrm{C}$ for $10 \mathrm{~min}$. The amplified products were detected through $1 \%$ gel electrophoresis and purified using agarose gel DNA purification Kit Ver. 4.0. The purified product was cloned into the pMD18-T vector and then transformed into Escherichia coli DH5a. Positive clones were selected and sent to Shanghai Sangon Biotechnology Company for sequencing.

\section{Bioinformatic analysis of CnFPPS}

Gene sequencing was performed on the http://www.ncbi. com (NCBI) website using the BLAST tool to determine the similarity of the CnFPPS nucleotide sequence with other plant FPPS nucleotide sequences. The open reading frame (ORF) of the CnFPPS gene was predicted, and CnFPPS protein sequence was compared with other homology of plant FPPS protein sequences using Vector NTI 11.5. The calculated pI and molecular weight of the CnFPPS protein were computed using the software of Compute $\mathrm{pI} / \mathrm{Mw}$ Tool at http://web. expasy.org/compute_pi/. Multiple sequence alignments were performed using the software Vector NTI 11.5 program. Phylogenetic analysis of CnFPPS protein sequence and other FPPS protein sequences from other plants was aligned with CLUSTALX 2, and subsequently, a phylogenetic tree was constructed through the neighbor-joining (NJ) method with MEGA 6 software.

Quantitative Real-time Polymerase Chain Reaction (qRT$P C R)$ analysis

Total RNA was extracted from roots, stems, leaves and flowers of $C$. nobile and reverse transcribed into cDNA. The

\begin{tabular}{cc}
\hline Primer & Sequences $\left(5^{\prime}-3^{\prime}\right)$ \\
\hline Upstream primer of CnFPPS gene $(\mathrm{G} 1)$ & AAAGAAGCGATACATCACTGAC \\
Downstream primer of CnFPPS gene $(\mathrm{G} 2)$ & CCACTCGCAAGACTCAAAATCAG \\
Upstream primer of qRT-PCR $(\mathrm{R} 1)$ & GTTGCCCTCTGCGTGTATGAGACTC \\
Downstream primer of qRT-PCR $(\mathrm{R} 2)$ & GATTTTCTTTCATCCGCTCTTGG \\
Upstream primer of 18S RNA $(\mathrm{S} 1)$ & AACGAGCGTCGAGTGGATTAA \\
Downstream primer of $18 \mathrm{~S}$ RNA $(\mathrm{S} 2)$ & CCCATCGAAGGGACTCCTATT \\
\hline
\end{tabular}


360

quantitative real-time polymerase chain reaction (qRT-PCR) primers (Table 1) of the CnFPPS gene were designed according to the real-time quantitative PCR kit of AceQ $^{\circ}$ qPCR SYBR ${ }^{\circ}$ Green Master Mix (without ROX). The reference gene for the RT-PCR is $18 S \mathrm{RNA}$, and the upstream and downstream primers were $S 1$ and $S 2$, respectively (Table 1). qRT-PCR was performed on a Bio-Rad CFX Fluorescence Quantitative PCR instrument using the Vazyme AceQ ${ }^{\circ}$ PCR SYBR $^{\circ}$ Green Master Mix (Without ROX) kit instructions. The solution curve was added under the following conditions: 40 cycles of at $95^{\circ} \mathrm{C}$ for $30 \mathrm{~s}, 95^{\circ} \mathrm{C}$ for $5 \mathrm{~s}$, and $60^{\circ} \mathrm{C}$ for $30 \mathrm{~s}$. qRT-PCR data were technically replicated with error bars, representing mean $\pm S D(n=3)$. The relative expression fold of each sample was calculated by its $\mathrm{Ct}$ value normalized to the Ct-value of reference gene using the $2^{-\Delta \Delta \mathrm{Ct}}$ method (Schmittgen et al., 2008).

\section{Results}

Cloning and sequence analysis of CnFPPS

A full-length cDNA of CnFPPS was 1239 bp long and contained a 1029 bp ORF encoding 342 amino acid proteins (Fig. 1). The results of BLASTN analysis at NCBI site showed that the cDNA sequence of CnFPPS had a high similarity to those of other FPPS genes. Collectively, these results indicated that the gene we cloned is the cDNA sequence of CnFPPS. Therefore, this gene was designated as CnFPPS (GenBank Accession No. KY432438).

\section{Characterization of the deduced CnFPPS protein}

The theoretical molecular weight and $\mathrm{p} I$ of the deduced CnFPPS protein were $39.38 \mathrm{KDa}$ and 5.59, respectively.

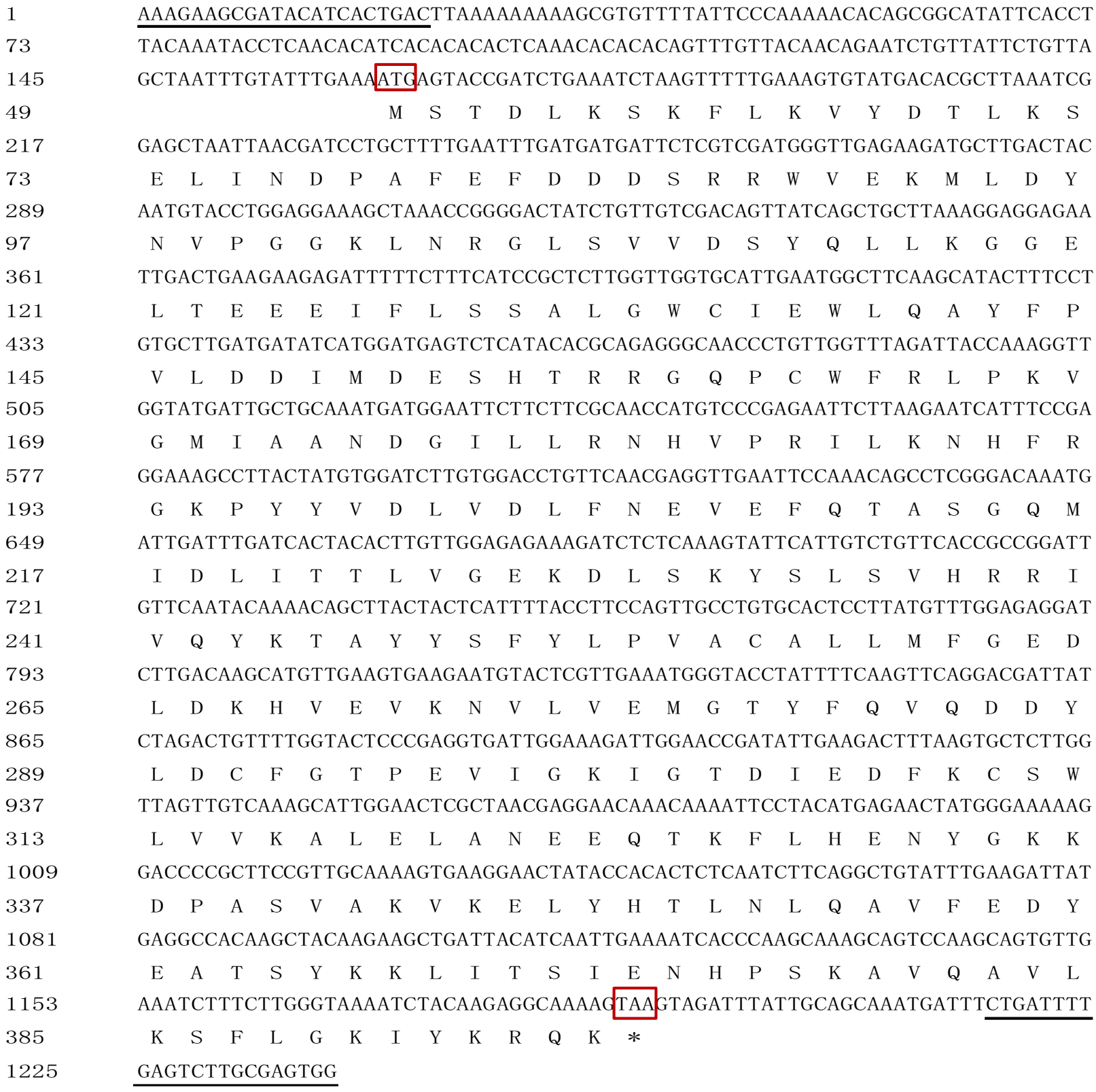

Fig. 1. Nucleotide sequence and deduced amino acid sequence of CnFPPS. The initial codon and the stop codon are highlighted in red square box; the primer sequences are underlined 
Table 2. Nucleotide sequence of CnFPPS similarity to the FPPSs of other plant species

\begin{tabular}{|c|c|c|c|}
\hline Species & GenBank Accession No. & Identity (\%) & E-value \\
\hline Achillea asiatica & JX424551.1 & $93 \%$ & 0.0 \\
\hline Tanacetum coccineum & JX424559.1 & $95 \%$ & 0.0 \\
\hline Leucanthemum vulgare & JX424557.1 & $94 \%$ & 0.0 \\
\hline Matricaria chamomilla & KJ130321.1 & $96 \%$ & 0.0 \\
\hline Artemisia annua & U36376.1 & $92 \%$ & 0.0 \\
\hline Artemisia tridentata & AY308477.1 & $93 \%$ & 0.0 \\
\hline Aster ageratoides & JX424562.1 & $88 \%$ & 0.0 \\
\hline Taraxacum kok-saghyz & KJ558350.1 & $87 \%$ & 0.0 \\
\hline Leibnitzia anandria & JX424569.1 & $86 \%$ & 0.0 \\
\hline
\end{tabular}

\begin{tabular}{|c|c|c|}
\hline $\begin{array}{l}\text { FPPS } \\
\text { aPPS } \\
\text { aPPS } \\
\text { FPPS } \\
\text { aFPPS } \\
\text { FPPS } \\
\text { aFPPS } \\
\text { FPPS }\end{array}$ & $\begin{array}{l}\text { (1) } \\
\text { (1) } \\
\text { (1) } \\
(1) \\
(1) \\
(1) \\
(1) \\
(1)\end{array}$ & 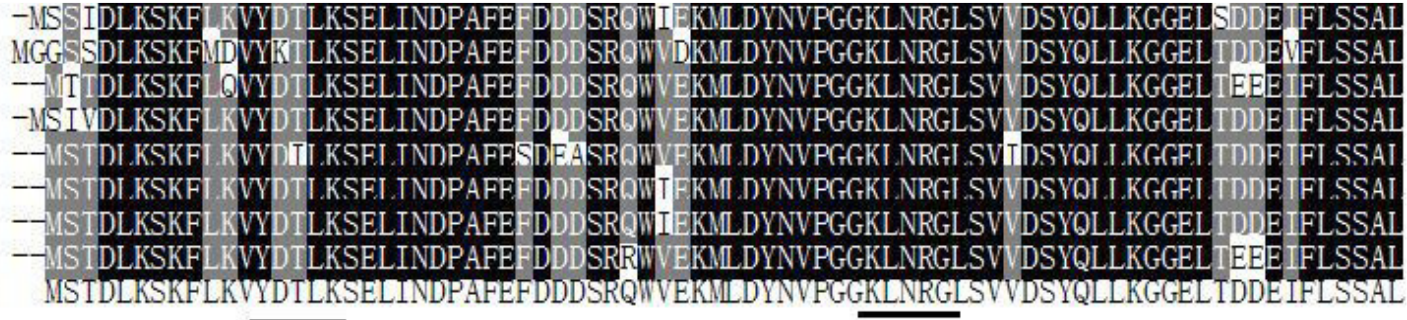 \\
\hline $\begin{array}{l}\text { PPS } \\
\text { PPS } \\
\text { PPS } \\
\text { PPS } \\
\text { PPS } \\
\text { PPS }\end{array}$ & $\begin{array}{l}(81) \\
(79) \\
(80) \\
(79) \\
(79) \\
(79) \\
(79) \\
(81)\end{array}$ & 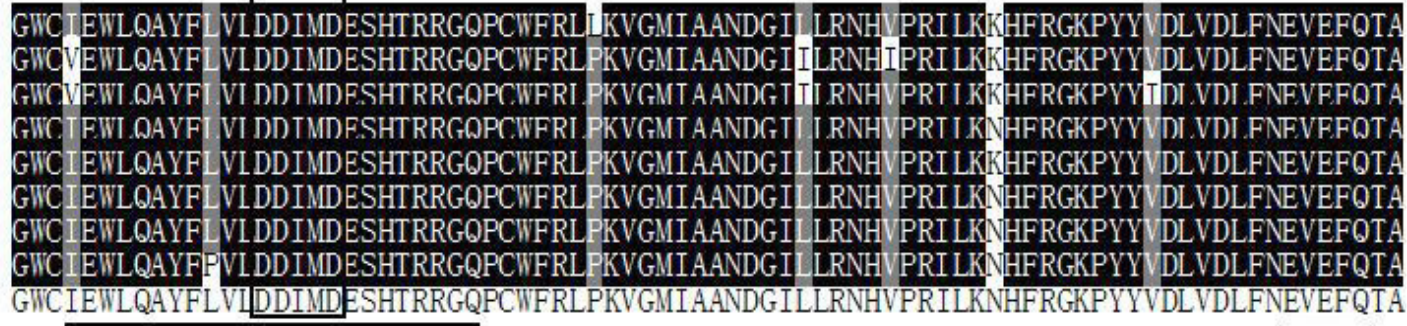 \\
\hline $\begin{array}{l}\text { PS } \\
\text { PPS }\end{array}$ & $\begin{array}{l}\text { 51) } \\
\text { 59) } \\
\text { 50) } \\
\text { 59) } \\
\text { 59) } \\
\text { 59) } \\
\text { 59) } \\
\text { 51) }\end{array}$ & 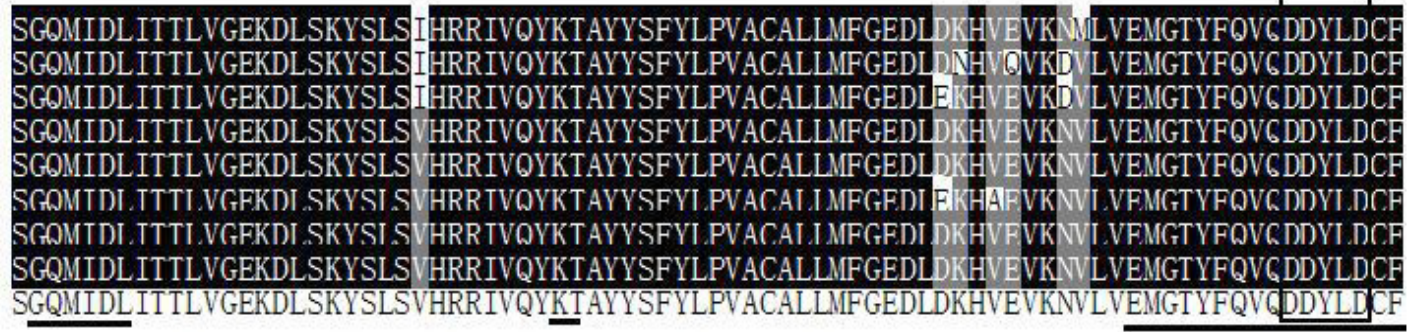 \\
\hline & $\begin{array}{l}\text { 40) } \\
\text { 41) } \\
\text { 39) } \\
\text { 40) } \\
\text { 39) } \\
\text { 39) } \\
\text { 39) } \\
\text { 39) } \\
\text { 41) }\end{array}$ & 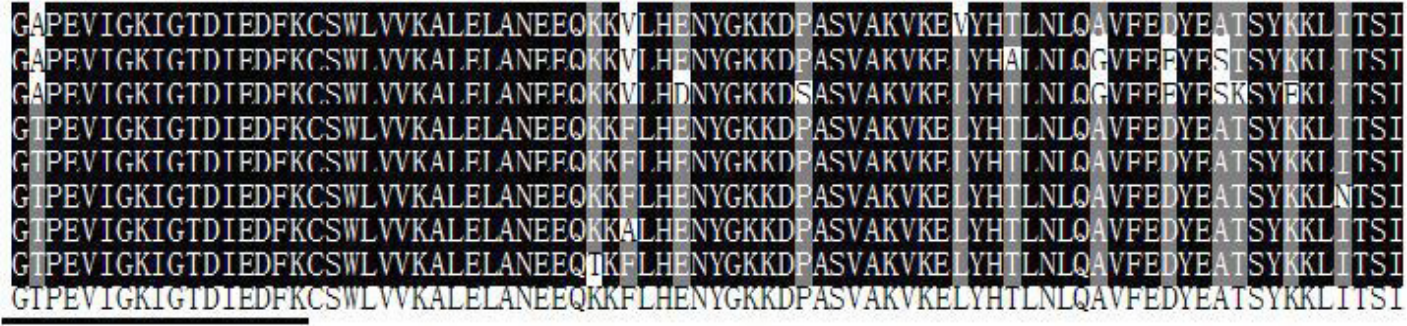 \\
\hline & $\begin{array}{l}(320) \\
(321) \\
(319) \\
(320) \\
(319) \\
(319) \\
(319) \\
(319)\end{array}$ & 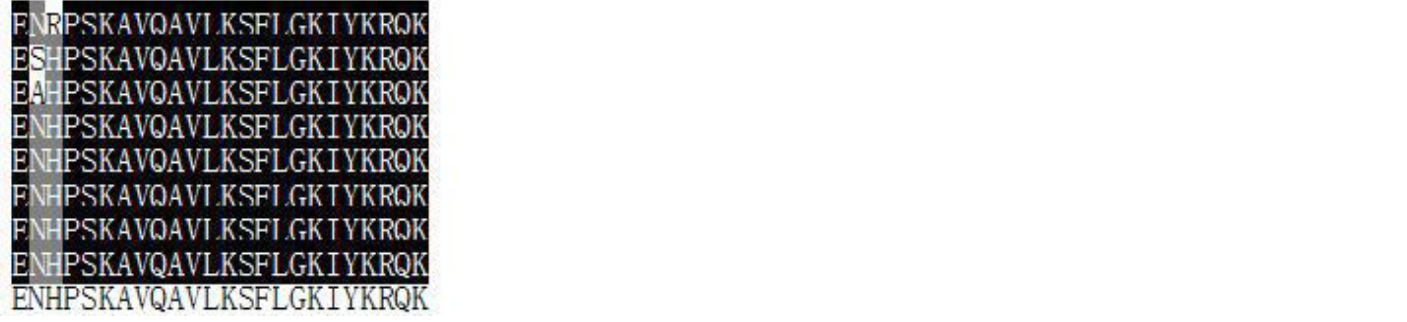 \\
\hline
\end{tabular}

Fig. 2. Similarity analysis of CnFPPS-coding protein and other known FPPS proteins. TcFPPS , T. coccineum; McFPPS , $M$. chamomilla; AcaFPPS, A. asiatica; LvFPPS, L. vulgare; AraFPPS, A. annua; TkFPPS, T. kok-saghyz; AsaFPPS, A. ageratoides; CnFPPS, C. nobile. White on black portion represents exactly the same, white on grey represents a conservative district. Five conserved domains are underlined, which are the substrate binding pocket, aspartate-rich regions 1 , catalytic site, substrate- $\mathrm{Mg}^{2+}$ binding site and aspartate-rich regions 2 . Two aspartate-rich regions highlighted in square box 


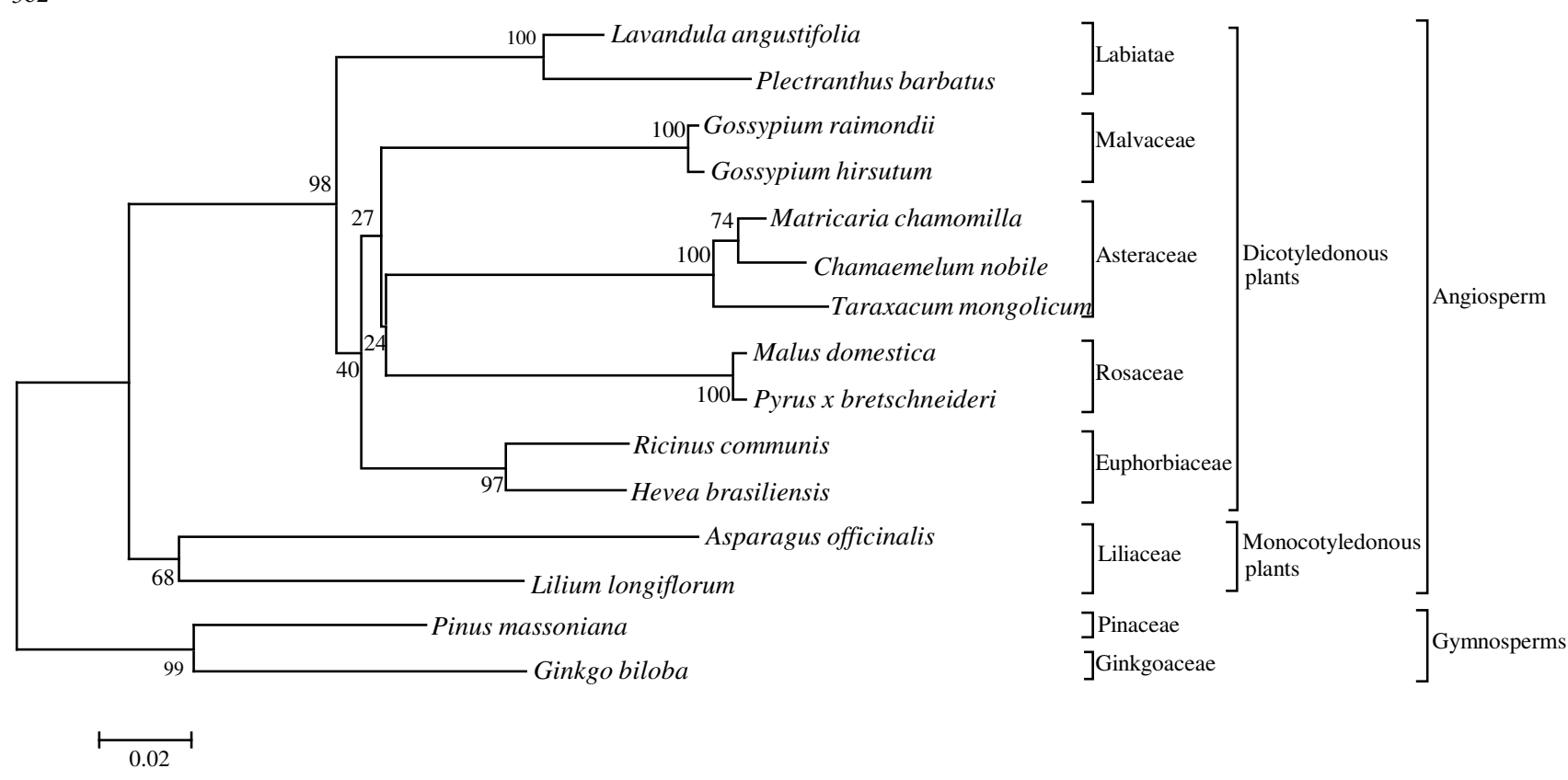

Fig. 3. Phylogenetic tree of genes in plant FPPS family

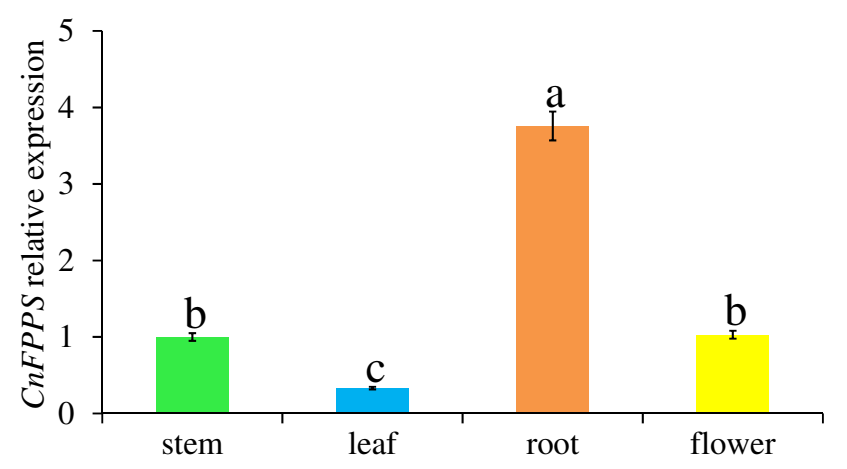

Fig. 4. Expression pattern of CnFPPS among different tissues of $C$. nobile

The nucleotide sequence of CnFPPS had a similarity of more than $85 \%$ to those with other FPPS genes (Table 2 ), indicating that the gene we cloned is a member of the FPPS gene family. Multiple sequence alignments revealed that CnFPPS had high homologous to FPPSs from other species. The CnFPPS protein showed 98\%, 98\%, 97\%, 97\%, 95\%, 94\%, and $92 \%$, similarity to the counterparts of Tanacetum coccineum, M. chamomilla, Acbillea asiatica, A. annua, Leucanthemum vulgare, Taraxacum koksaghyz, and Aster ageratoides, respectively. In addition, CnFPPS contained five conservative domains, namely, a substrate binding pocket, a substrate- $\mathrm{Mg}^{2+}$ binding site, a catalytic site, and aspartate-rich regions 1 and 2 (Fig. 2).

\section{Analysis of the molecular evolution of CnFPPS}

To investigate the evolutionary relationships in CnFPPS and FPPS proteins from other species, we selected the typical FPPS proteins from the GenBank. We constructed a phylogenetic tree using the software MEGA 6.0 with NJ method. As shown in Fig. 3, FPPS phylogenetic tree is divided into two broad clades: one is angiosperm, including Labiatae, Euphorbiaceae, Malvaceae, Rosaceae and Asteraceae of Dicotyledoneae and Liliaceae of Monocotyledoneae; the other is gymnosperm, including Ginkgoaceae and Pinaceae. The CnFPPS protein has the closest relationship to McFPPS of $M$. chamomilla and TmFPPS of Taraxacu mmongolicum. They both belong to the Asteraceae family, indicating that the genetic relationship between the FPPSs gene of the Asteraceae family was close. Meanwhile, Asteraceae FPPS has the furthest relationship with Liliaceae, Ginkgoaceae, and Pinaceae. Liliaceae belongs to monocotyledonous plants, whereas Ginkgoaceae and Pinaceae belong to gymnosperms.

\section{Expression analysis of FPPS in different tissues of C. nobile}

qRT-PCR analysis showed that the CnFPPS gene was expressed in the roots, stems, leaves and flowers of $C$. nobile. However, CnFPPS gene expression was variuos in different tissues (Fig. 4), with the highest expression level in roots, followed by flowers and stems, and the lowest expression level in leaves.

\section{Discussion}

In recent years, remarkable progress has been made in the molecular regulation of sesquiterpenoid biosynthesis in plants (Yu et al., 2009; Degenhardt et al., 2009). FPPS is a key enzyme in the sesquiterpenoid biosynthetic pathway, and its activity is closely related to the accumulation of subsequent products. It has been studied to increase the content of active ingredients in plants by overexpressing the FPPS gene. For example, in $A$. annua, overexpressed the FPPS gene of Asian cotton and A. annua, could improve content of artemisinin (Han et al., 2006; Banyai $e t$ al., 2010). The amount of sterol and triterpene substances was increased with the increased expression of 
ginseng FPPS gene in Centella asiatica (Kim et al., 2010). Thence, with the isolation and identification of secondary metabolite synthesis enzymes in the metabolic pathways of plants, the use of genetic engineering to alter the expression of enzymes and thus change the yield and species of secondary metabolism has become a new method to improve plant quality (Ren et al., 2005). Therefore, the overexpression of the CnFPPS gene in C. nobile is hypothesized to increase the sesquiterpene content of $C$. nobile.

In this study, multiple alignments showed that the deduced CnFPPS sequence exhibited high similarity to FPPS proteins from other plants. The FPPS gene of $C$. nobile may play similar biological functions as that of FPPS of other plants and participate in terpenoid biosynthesis in $C$. nobile. The phylogenetic tree indicated that CnFPPS has a distinct and long-standing relationship with the FPPSs from other Asteraceae plants. Moreover, protein motif analysis showed that the CnFPPS contained five conserved domains in the FPPS protein family, namely, substrate binding pocket, substrate- $\mathrm{Mg}^{2+}$ binding site, catalytic site, and aspartate-rich regions 1 and 2. Almost all TPSs have been reported to contain a typical conserved sequence of the sesquiterpene synthase gene family, that is, the aspartic acid-enriched motif, which is thought to have an important effect of binding to metal ions and guide substrate catalysis (Christianson et al., 2006). And the motif is thought to have an important effect of binding to metal ions and guide substrate catalysis (Christianson et al., 2006), suggesting that CnFPPS plays an important role in the production of sesquiterpenoids in C. nobile.

Several studies showed that the expression pattern of FPPS in plant tissues significantly varies across different plants. For example, FPPS is strongly expressed in leaf and root, moderately expressed in stem, and weakly expressed in the stem of G. biloba (Wang et al., 2004). FPPS genes are commonly found in tomato plants and are regulated during development (Gaffe et al., 2000). FPPS was mainly expressed in the tubular flowers, moderately expressed in the ligulate flowers and leaves, and least expressed in the root of M. chamomilla ( $\mathrm{Su}$ et al., 2015). The expression pattern of CnFPPS in C. nobile revealed that the gene was expressed in all tissues but is expressed at a high level in the root, followed by that in flower and stem, and expression level was lowest in leaf. $C$. nobile is rich in active components and is among the most popular herbs since ancient times. The flowers of chamomile plants are commonly used for medicinal purposes. A recent chemical analysis of chamomile has shown that the content of sesquiterpene in flowers is highest and chamazulene is the most abundant sesquiterpene compound (Farhoudi et al., 2013). Given the high number of members of the FPPS gene family, the CnFPPS cloned in this study is only a part of its family and does not represent the function of the entire FPPS family in $C$. nobile. To better analyze the function of FPPS gene in C. nobile and determine whether the content of terpene compounds in C. nobile can be improved by transgenic technology of the gene obtained by cloning, further studies about gene cloning and functional analysis of other members of FPPS gene family of $C$. nobile are needed. Such studies will lay the foundation for studying the molecular mechanism of the biosynthesis of terrestrial compounds of $C$. nobile.

\section{Acknowledgements}

This work was supported by National Natural Science Foundation of China (No. 31400603).

\section{References}

Attucci S, Aitken SM, Ibrahim RK, Gulick PJ (1995). A cDNA encoding farnesyl pyrophosphate synthase in white lupin. Plant Physiology 108(2):835-836.

Banyai W, Kirdmanee C, Mii M, Supaibulwatana K (2010). Overexpression of farnesyl pyrophosphate synthase (FPS) gene affected artemisinin content and growth of Artemisia annua L. Plant Cell, Tissue and Organ Culture 103(2):255-265.

Chang J, Ning Y, Xu F, Cheng S, Li X (2015). Research advance of 3hydroxy-3-methylglutaryl-coenzyme a synthase in plant isoprenoid biosynthesis. Journal of Animal \& Plant Sciences 25(5):1441-1450.

Chappell J (1995). Biochemistry and molecular biology of the isoprenoid biosynthetic pathway in plants. Annual Review of Plant Biology 46(1):521-547.

Christianson DW (2006). Structural biology and chemistry of the terpenoid cyclases. Chemical Reviews 106(8):3412-3442.

Cornish K (1993). The separate roles of plant cis and trans prenyl transferases in cis-1,4-polyisoprene biosynthesis. European Journal of Biochemistry 218(1):267-271.

Degenhardt J, Köllner TG, Gershenzon J (2009). Monoterpene and sesquiterpene synthases and the origin of terpene skeletal diversity in plants. Phytochemistry70(15-16):1621-1637.

Delourme D, LacrouteF, Karst F (1994). Cloning of an Arabidopsis thaliana cDNA coding for farnesyl diphosphate synthase by functional complementation in yeast. Plant Molecular Biology 26(6):1867-1873.

Enfissi E, Fraser PD, Lois LM, Boronat A, Schuch W, Bramley PM (2005). Metabolic engineering of the mevalonate and non-mevalonate isopentenyl diphosphate-forming pathways for the production of health-promoting isoprenoids in tomato. Plant Biotechnology Journal $3(1): 17-27$.

Farhoudi R (2013). Chemical constituents and antioxidant properties of Matricaria recutita and Chamaemelum nobile essential oil growing wild in the south west of Iran. Journal of Essential Oil Bearing Plants 16(4):531-537.

Fauconnier ML, Jaziri M, Homes J, Shimomura K, Marlier M (1996). Anthemis nobilis L. (Roman chamomile): In vitro culture, micropropagation, and the production of essential oils. Medicinal \& Aromatic Plants IXpp 16-37.

Gaffe J, Bru JP, Causse M, Vidal A, Stamitti-Bert L, Carde JP, Gallusci P (2000).LEFPS1, a tomato farnesyl pyrophosphate gene highly expressed duringearly fruit development. Plant Physiology 123(4):1351-1362.

Han JL, Liu BY, Ye HC, Wang H, Li ZQ, Li GF (2006). Effects of overexpression of the endogenous farnesyl diphosphate synthase on the artemisinin content in Artemisia annua L. Journal of Integrative Plant Biology 48(4):482-487.

He YF, Gao W, Liu TS, Li WY, Huang LQ (2011). Research advances of diterpene synthase. Acta PharmaceuticaSinica(9):1019-1025. 
364

Kim OT, Kim SH, Ohyama K, Muranaka T, Choi YE, Lee HY,Hwang B (2010). Upregulation of phytosterol and triterpene biosynthesis in Centella asiatica hairy roots overexpressed ginseng farnesyl diphosphate synthase. Plant Cell Reports 29(4):403-411.

Lange BM, Rujan T, Martin W, Croteau R (2000). Isoprenoid biosynthesis: the evolution of two ancient and distinct pathways across genomes. Proceedings of the National Academy of Sciences USA 97(24):1317213177.

Lange BM, Wildung MR, Stauber EJ, Sanchez C, Pouchnik D, Croteau R (2000). Probing essential oil biosynthesis and secretion by functional evaluation of expressed sequence tags from mint glandular trichomes. Proceedings of the National Academy of Sciences USA 97(6):2934 2939.

Liu C, Meng Y, Hou S, Chen X (1997). Cloning and sequencing of a cDNA encoding farnesyl pyrophosphate synthase from, Gossypium arboreum and its expression pattern in the developing seeds of, Gossypium hirsutum cv. 'Sumian-6. Acta Botanica Sinica 40(8):703710.

Liu Z, Li X, Simoneau AR, Jafari M, Zi X (2006). Isoprenoid biosynthesis in plants: pathways, genes, regulation and metabolic engineering. Journal of Biological Sciences 6(1):371-374.

Ma CM, Winsor L, Daneshtalab M (2007). Quantification of spiroether isomers and herniarin of different parts of Matricaria matricarioides and flowers of Chamaemelum nobile. Phytochemical Analysis 18(1):42-49.

Ma ZZ, Pang XQ, Sheng R, Pei XI, Wang QY, Xie T, Yin XP (2015). Research advances of key enzymes in the biosynthes is pathways of isoprenoids. Journal of Hangzhou Normal University (Natural Science Edition) 14(6):608-615.

Matsushita Y, Kang W, Charlwood BV (1996). Cloning and analysis of a cDNA encoding farnesyl diphosphate synthase from Artemisia annua. Gene 172(2):207-209.
Ohto C, Ishida C, Nakane H, Muramatsu M, Nishino T, Obata S (1999). A thermophilic cyanobacterium Synechococcus elongatus has three different Class I prenyltransferase genes. Plant Molecular Biology 40(2):307-321.

Ren Y, Lu G, Cao JS, Li YJ (2005). Improving flavor by metabolic engineering of the terpenoid pathway in crop plants. Chinese Journal of Cell Biology 27(3):319-324.

Schmittgen TD, Livak KJ (2008). Analyzing real-time PCR data by the comparative CT method. Nature Protocols3(6):1101-1108.

Sharkey TD, Gray DW, Pell HK, Breneman SR, Topper L (2013). Isoprene synthase genes form a monophyletic clade of acyclic terpene synthases in the TPS-B terpene synthase family. Evolution 67(4):10261040.

SrivastavaJK, ShankarE, GuptaS(2010). Chamomile: A herbal medicine of the past with bright future. Molecular Medicine Reports 3(6):895-901.

SuSS,Zhang HM, LiuXY, Pan GF, LingSP,ZhangXS, YangXM, Tai YL, Yuan Y (2015). Cloning and characterization of a farnesyl pyrophosphate synthase from Matricaria recutita $\mathrm{L}$. and its upregulation by methyl jasmonate. Genetics \& Molecular Research 14(1):349-361.

Vranová E, Coman D, Gruissem W (2012). Structure and dynamics of the isoprenoid pathway network. Molecular Plant 5(2):318-333.

Wang P, Liao Z, Guo L, Li W, Chen M, Pi Y, Tang K (2004). Cloning and functional analysis of a cDNA encoding Ginkgo biloba farnesyl diphosphate synthase. Molecules \& Cells 18(2):150-156.

Xiao LYD (2003). Antipruritic effects of Matricaria extracts. International Journal of Traditional Chinese Medicine25(3):173-174.

Yu F, Utsumi R (2009). Diversity, regulation, and genetic manipulation of plant mono-and sesquiterpenoid biosynthesis. Cellular \& Molecular Life Sciences 66(18):3043-3052.

Yue YC, Fan YP (2011). The terpene synthases and regulation of terpene metabolism in plants. Acta Horticulturae Sinica 38(2):379-388. 